\title{
To the issue of energy efficiency of using frequency-controlled centrifugal pump units
}

\author{
Alexey Dmitriev ${ }^{1 *}$ and Vyacheslav Gerasimov ${ }^{1}$ \\ ${ }^{1}$ Tyumen Industrial University, Volodarskogo str., 38, Tyumen, 625000, Russia
}

\begin{abstract}
The expediency of using a variable frequency drive for pumping units was repeatedly proved both from the point of view of the economic component and the technological one. However, the construction of automatic control systems for the operating parameters of pumping stations is mainly based on maintaining the pressure setpoint and does not include monitoring the efficiency of the aggregates working in the group and, the more so, its regulation. In this paper, an algorithm is developed for the energy efficient management of centrifugal pump units, which allows not only maintaining the pressure setpoint, but also optimizing their efficiency.
\end{abstract}

\section{Introduction}

Energy saving at pumping stations is achieved by using frequency converters to regulate the rotor speed of pump units. Such a technical solution is more economical in comparison with such traditional methods of controlling the delivery of pumping stations as throttling and bypassing. Multi-aggregate pumping stations with a parallel way of pumps connection use cascade frequency control systems for pump units. Quite often, regardless of the number of pump units, only one is always adjustable. An adjustable pump unit is called the master pump. The peculiarity of this method of regulation is that in order to maintain the setpoint of pressure a switching process of pump units occurs. The process proceeds by disconnection of one pump unit from the frequency converter and connecting it directly to the network with the practically simultaneous connection of the frequency converter to another centrifugal pump unit. This method of regulation is quite effective, but not enough energy efficient, since the automatic control system can maintain the pressure at a given point of the hydraulic network with a sufficient degree of accuracy, but the efficiency of the pumping units is not monitored in any way.

\section{Method and Result}

Figure 1 shows the situation with the non-optimal loading of pump units. This situation is typical for systems of automatic regulation of pipeline pressure. For the generality of the results obtained, the calculation is made in relative units. The rated values of the variables

\footnotetext{
* Corresponding author: alextmn86@mail.ru
} 
are taken as base values. The calculation is valid for centrifugal pumps that have gentle pressure characteristics [1].

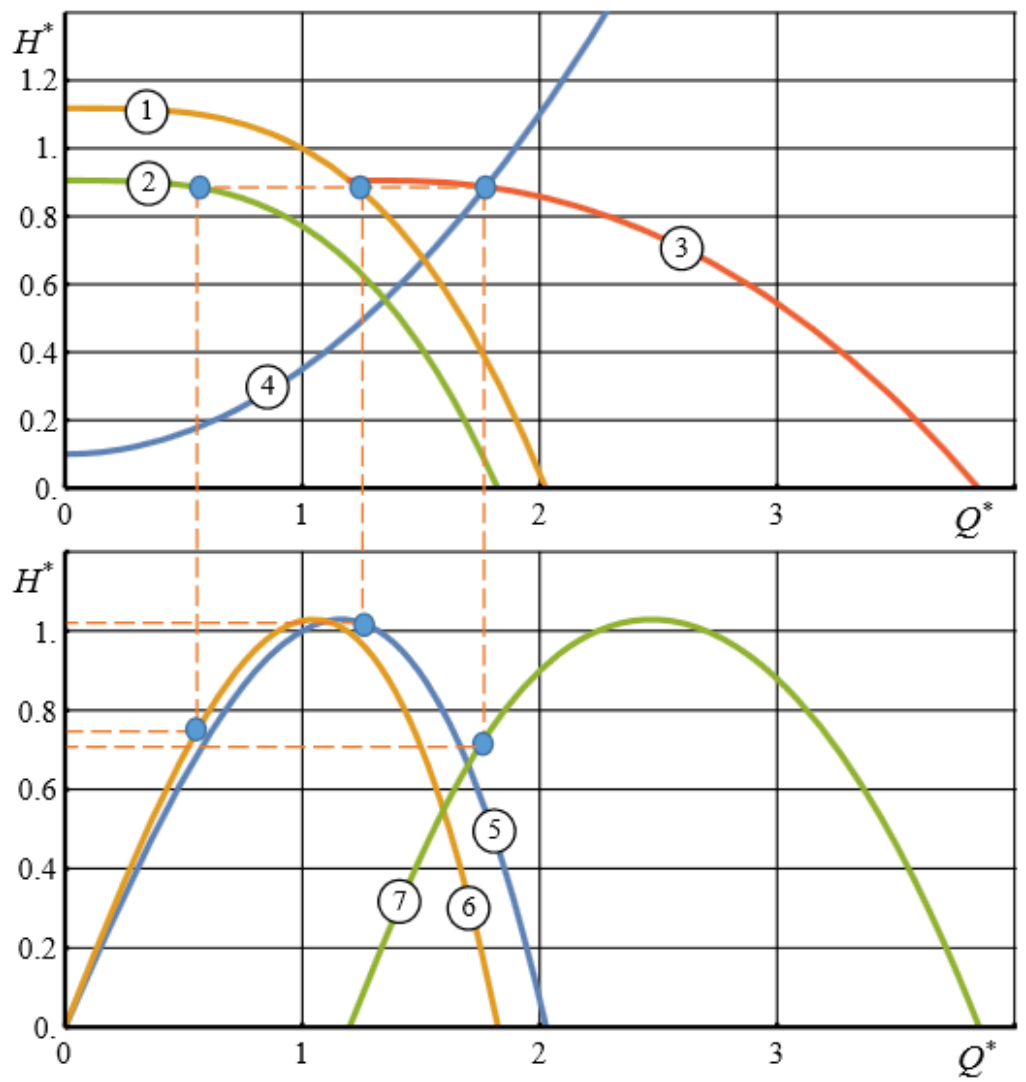

Fig. 1. Family of hydraulic characteristics of pump units.

1 - Pressure characteristic of pump unit, operating with frequency $\alpha_{1} ; 2$ - Pressure characteristic of pump unit, operating with frequency $\alpha_{2} ; 3$ - Joint pressure characteristic of two pump units; 4 Pressure characteristic of the pipeline network; 5 - Efficiency of pump unit, working with frequency $\alpha_{1} ; 6$ - Efficiency of pump unit, operating with frequency $\alpha_{2} ; 7$ - Average efficiency of the group.

The hydraulic characteristics of frequency-controlled centrifugal pump units in relative units are described by approximating polynomials of degree 3 :

$$
\begin{gathered}
H^{*}\left(Q^{*}\right)=a_{0}^{*} \cdot \alpha_{i}^{2}-a_{1}^{*} \cdot \alpha_{i} \cdot Q^{*}+a_{2}^{*} \cdot Q^{* 2}-a_{3}^{*} \cdot \frac{1}{\alpha_{i}} \cdot Q^{* 3} ; \\
\eta^{*}\left(Q^{*}\right)=b_{1}^{*} \cdot \frac{1}{\alpha_{i}} \cdot Q^{*}-b_{2}^{*} \cdot \frac{1}{\alpha_{i}^{2}} \cdot Q^{* 2}-b_{3}^{*} \cdot \frac{1}{\alpha_{i}^{3}} \cdot Q^{* 3},
\end{gathered}
$$

$a_{1}^{*}$ - relative rotational speed of the rotor of $i$-pump; $H^{*}$ - pressure of pump unit; $Q^{*}$ - flaw of a pump unit; $a_{0}^{*}, a_{1}^{*}, a_{2}^{*}, a_{3}^{*}$ - coefficients of approximation of the pump discharge characteristic at the nominal rotor speed; $b_{1}^{*}, b_{2}^{*}, b_{3}^{*}$ - coefficients of approximation of the pump efficiency at the nominal rotor speed. 
The values of the approximation coefficients are presented in Table 1.

Table 1. The values of the approximation coefficients.

\begin{tabular}{|c|c|c|c|}
\hline Name & Value & Name & Value \\
\hline$a_{0}^{*}$ & 1,118 & $b_{1}^{*}$ & 1,359 \\
\hline$a_{1}^{*}$ & 0,007 & $b_{2}^{*}$ & 0,054 \\
\hline$a_{2}^{*}$ & 0,042 & $b_{3}^{*}$ & 0,304 \\
\hline$a_{3}^{*}$ & 0,154 & - & - \\
\hline
\end{tabular}

The average efficiency in relative units for a group of parallel connected pump units is determined according to the expression [2]:

$$
\eta^{*} \quad\left(Q_{\Sigma}^{*}\right)=\frac{Q_{\Sigma}^{*}}{\sum_{i=1}^{n} Q_{i}^{*} / \eta_{i}^{*}},
$$

$Q_{i}^{*}, \eta_{i}^{*}$ - supply and efficiency of the $i$-pump unit at the current value of the pressure in the common receiving manifold, respectively; $Q_{\Sigma}^{*}-$ total flow of the pump group.

It is the average efficiency of a group of centrifugal pumping units that makes sense to be taken as an objective function and optimized using an evolutionary algorithm based on the following conditions:

$$
\begin{aligned}
& \alpha_{i}=\text { var; } \\
& 0 \leq \alpha_{i} \leq \alpha_{\max } \\
& \sum_{i=1}^{n} Q_{i}^{*}=Q_{\Sigma}^{*} ; \\
& Q_{i}^{*} \geq 0 ; \\
& H_{i}^{*}\left(Q_{i}^{*}\right)=H_{S}^{*} .
\end{aligned}
$$

- The current pressure value is determined in the common collector of the pump units due to feedback from the pressure sensor;

- The value of the total flow and flow of each pump unit is determined by the installation of flow sensors on the lines extending from the common high-pressure collector, and also in the intervals between the check valves of the pump units and the intake manifold;

- The current pressure value is determined by means of pressure sensors installed both on the common receiving manifold and at the outlet of each pump unit. This fact allows to precisely control and regulate the pressure in the pipeline.

- The current pressure value coincides with the pressure setpoint $H_{S}^{*}$, since it is assumed that the automatic pressure regulation system has been operated. Then there is the redistribution of the loading of the pump units in accordance with condition $\eta^{*} \quad\left(Q_{\Sigma}^{*}\right) \rightarrow \max$.

The system of automatic regulation of pressure works according to the developed algorithm presented in Figure 2. 


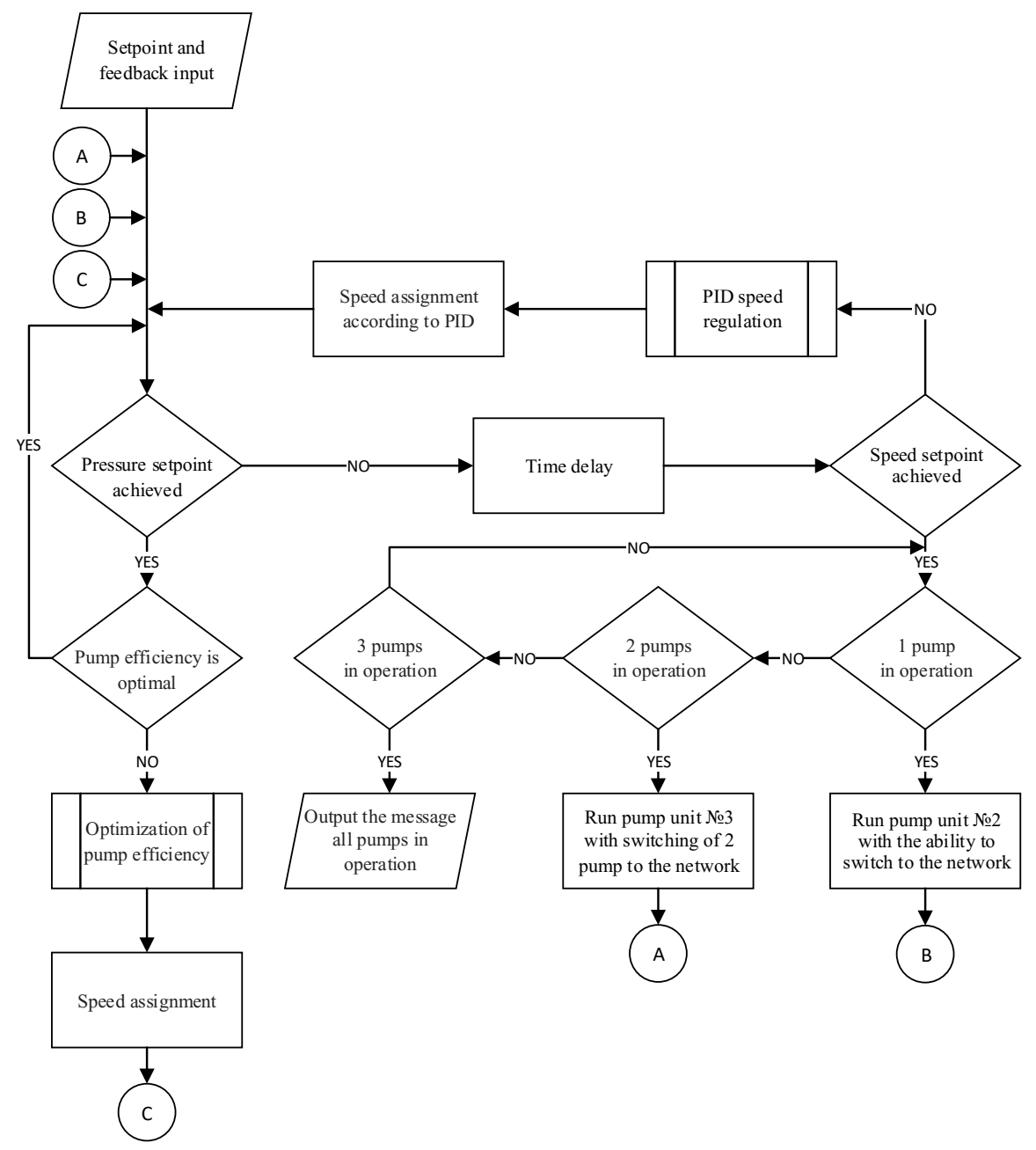

Fig. 2. The developed algorithm for the automatic control system of the pump station pressure.

This algorithm allows to carry out the control process if there is one adjustable pump unit in the group, as well as several adjustable ones. In the presence of one adjustable pump unit, the process of redistribution of the pump units does not take exist, but they are switched from the frequency converter to the electric network. However, in most cases, in order to achieve optimal energy utilization of a single regulated pumping unit, it is not enough.

The developed algorithm assumes realization of the closed system of automatic pressure regulation of the pump unit with the subordinate regulation and consequent correction of the parameters. The system of subordinate regulation in the general case consists of a series of loops, the number of which is equal to the number of controlled variables or to the number of large system time constants to be compensated, each internal control loop being subordinate to the next external loop. This subordination is expressed in the fact that the set value of the adjustable variable of any internal loop is determined by the output signal of the regulator of the external loop As a result, all internal loops work as subordinate to the control problem of the output coordinate of the system. Each loop is built on the principle of regulation by deviation and has its usually hard negative feedback on the regulated variable and its regulator. For each external loop, the internal loop or several internal loops 
are included in the control object. The system of automatic regulation of the pressure of the pump set includes a pressure control loop and a speed control loop with the corresponding regulators. The minor loop is the speed control loop of the electric motor. The main control loop is the pressure control loop created by the pump unit at a given point in the pipeline network. It is necessary to take into account the fact that at the moment of starting of the pump unit, there will be a significant difference between the pressure setpoint and the current pressure value at a given point in the pipeline. This difference is a pressure error signal which, after appropriate correction, is supplied as a reference signal to the speed controller, and causes the formation of an incorrect reference signal via the voltage and frequency control channels of the frequency converter. As a result, there is a sharp increase in the electromagnetic moment of the motor, which is a very undesirable phenomenon. It is necessary to optimize the system by the adjustment of the value and the rate of increase in the output signal of the pressure regulator in order to avoid large values of the speed reference signal. A similar effect can also be achieved by using variable feedback, tuned to work after the pump unit start-up.

\section{Conclusions}

1. Increasing of the number of measuring instruments provides the possibility of implementing an accurate control algorithm for a group of pump units. In particular, the installation of flow meters between the check valves and the common receiver collector allows obtaining accurate data on the flow of each parallel-connected centrifugal pump unit in the group. This fact allows, under the condition of known approximating polynomials, to determine the efficiency of each pump unit.

2. Increasing the upper limit of the speed range of the pump units allows to reduce the energy losses by not requiring parallel operation of the next pump unit from the group. This advantage is especially pronounced in the boundary conditions, when the operating pump set at the rated speed cannot cope with maintaining the required pressure, but the connection of the next pump unit is not economically beneficial due to the low efficiency of the latter.

3. Low efficiency is due to the uneven loading of pump units in parallel operation at different speeds. There is need to adjust the dead band of the pressure regulator to small deviations in the pressure setpoint to prevent frequent switching of the pump units due to fluctuations in the setpoint. The ability to regulate the speed of the pump set above the nominal allows one to regulate with one unit without the need to connect the next unit and there is no need to adjust the dead band.

4. The developed algorithm of controlling of centrifugal pump units ensures operation of each adjustable pump unit in the operating range of flow, which leads to an increase in the efficiency of the pumping station and significant energy savings.

\section{References}

1. A.K. Mikhaylov, V.V. Malyushenko Lopastnye nasosy. Teoriya, raschet $i$ konstruirovanie (2004)

2. O.A. Lysova, V.P. Frayshteter, Gornyy zhurnal. 82-91 (2014) 